Check for updates

Cite this: RSC Adv., 2019, 9, 26381

Received 24th June 2019

Accepted 4th August 2019

DOI: $10.1039 / c 9 r a 04737 a$

rsc.li/rsc-advances

\section{Applications and potential mechanisms of herbal medicines for rheumatoid arthritis treatment: a systematic review $\uparrow$}

\author{
Tai-ping Li, ${ }^{\text {ab }}$ Ai-hua Zhang, ${ }^{\mathrm{b}}$ Jian-hua Miao, ${ }^{\text {a Hui Sun, }}{ }^{\text {tb }}$ Guang-li Yan, ${ }^{\text {b }}$ \\ Fang-fang $\mathrm{Wu}^{\mathrm{ab}}$ and $\mathrm{Xi-jun} \mathrm{Wang} \mathrm{(D)} \mathrm{*ab}$
}

In this review, we systematically discuss the role of traditional Chinese medicine (TCM) in rheumatoid arthritis (RA) disease treatment. TCM classifies the subtypes of RA through its own theoretical method, which is beneficial for more accurate diagnosis and treatment with Chinese herbal medicines (CHMs) that are more suitable for different syndromes. TCM mainly uses a flexible combination of CHMs to play an important role in RA treatment. The main components of these extracts can be subdivided into alkaloids, flavonoids, triterpenes, saponins and other compounds. Using a platform of transgenic and induced arthritis models, we explore the potential mechanisms of TCM against RA with the help of omics analysis techniques and methods. These mechanisms are mainly CHM and its extracts can inhibit RA patients and experimental animal models, including synovitis, vascular proliferation and bone injury; this involves many biological signal exchange targets and pathways. In conclusion, the role of TCM in RA treatment mainly involves reducing the expression and secretion of pro-inflammatory factors, thus decreasing the degree of abnormal immune response.

\section{Introduction}

Rheumatoid arthritis (RA) is a long-term autoimmune disease; the joints of the affected population show symmetrical swelling and pain, and there is serious stiffness after rest. ${ }^{1}$ The pathogenesis may involve a combination of genetic and environmental factors, although the pathogenesis has not been shown, but most researchers are involved in studying joint injuries related to the inflammatory response, such as synovitis, pannus, cartilage erosion and other signaling pathways. ${ }^{2,3}$ RA can also cause many other diseases; ${ }^{4}$ the later stage of RA will lead to irreversible bone injury, which will lead to joint motion disorder, and many studies have found that patients with RA have an increased risk of other complications including myocardial infarctions, strokes, lung function issues and neurological abnormalities and depression. ${ }^{4,5}$ Severe cases lead to disability or even greater than expected mortality levels, ${ }^{6}$ and the result of unsatisfactory treatment is that the long RA

${ }^{a}$ National Engineering Laboratory for the Development of Southwestern Endangered Medicinal Materials, Guangxi Botanical Garden of Medicinal Plant, Nanning, Guangxi, China

${ }^{b}$ National Chinmedomics Research Center, Sino-America Chinmedomics Technology Collaboration Center, National TCM Key Laboratory of Serum Pharmacochemistry, Laboratory of Metabolomics, Department of Pharmaceutical Analysis, Heilongjiang University of Chinese Medicine, Heping Road 24, Harbin, China. E-mail: xijunwangls@126.com; Fax: +86-451-82110818; Tel: +86-451-82110818

$\dagger$ Electronic supplementary information (ESI) available. See DOI: 10.1039/c9ra04737a treatment process and lack of effective treatment can cause physical, mental and economic stress to the patient. ${ }^{7}$ RA affects about 24.5 million people as of $2015 .^{8}$ This is between 0.5 and $1 \%$ of adults in the developed world, with between 5 and 50 per 100000 people newly developing the condition each year. ${ }^{9}$ So far, the goal of RA treatment is to antagonize inflammatory reactions and relieve pain. ${ }^{\mathbf{1 4}}$ Along with NSAIDs, steroids and disease-modifying antirheumatic drugs (DMARDs), RA healthcare mainly relies on auxiliary equipment such as stents and splints to improve the overall function of the body, ${ }^{\mathbf{1 0}}$ and surgical treatment of joints in serious cases can inhibit the disease in time. However, long-term use of the above drugs will have adverse results, for example NSAIDs can lead to gastrointestinal bleeding, dyspepsia and other reactions. ${ }^{\mathbf{1 1}}$

Traditional Chinese medicine (TCM) has long since been applied, and was widely used in many places in East Asia at first. As a method of treatment and healthcare for diseases, it has become an integral part of complementary and alternative medicine approaches, and now it is also gradually being applied in various western countries. It is based on the theories of TCM for the treatment of numerous diseases, including the use of acupuncture and cupping; these methods involve the flexible use of Chinese herbal medicines (CHMs) and their related extracts. RA in the TCM classification system belongs to the "Bi syndrome" class, the earliest seen in the TCM treatise "Huang Di Nei Jing". ${ }^{12}$ Bi syndrome is due to a lack of qi and blood, caused by cold and humid heat invading the body as a result of 
issues with the catheter and meridians in blood circulation obstruction syndrome..$^{13,14}$

In this review, the application of TCM to RA related research is systematically introduced and discussed, including the treatment of RA in TCM, the representative compounds and proprietary Chinese medicines used, the active components contained in CHMs, and the biological carriers and dosages from pharmacodynamic research, on the basis of which the potential mechanisms of the TCM prevention and treatment of $\mathrm{RA}$, including the relevant genes, proteins, metabolites and pathways, are expounded.

\section{RA exposition based on TCM theory}

TCM adheres to "holistic concepts" and "dialectical treatments" for many diseases, that is, it is based on the unique signs of different patients, finally adopting personalized treatment. RA belongs to the Bi syndrome class in the field of TCM, which is characterized by the obstruction of meridians, ${ }^{15,16}$ and it can also be divided into cold, hot and defect syndrome characteristics.

The symptoms of hot are related to heat, swelling, and the inflammation of joints, and severe pain can generally be relieved by the cooling of the joints. Other symptoms include a red and yellow tongue; a fast pulse; a red face; constipation and darker urine; thirst; and irritability. ${ }^{17,18}$ Defect patterns usually occur in the late stage of the disease and are characterized by a deformity: the extension of the limb is suppressed. ${ }^{17}$ Defect symptoms combine cold and hot features. Patients with hot symptoms showed higher ACPA levels, more serious bone damage, higher levels of immune factors, increased expressions of genes related to the small $\mathrm{G}$ protein signaling pathway (TIAM1) and lipid metabolism (ALOX5), severe inflammatory reactions and high inflammatory activity in rheumatoid arthritis. ${ }^{18,19}$

Cold symptoms are described as an attack from an external "cold" pathogen algorithm, resulting in symptoms such as a cold feeling in the limbs and joints; stiffness or pain of the joints or muscles, which is relieved by the application of warmth and increases with the exposure of the patient to the cold; a local reduction in microcirculation; a thin white tongue; a strong and nervous pulse; a pale face; thin stools; a large amount of urine; and no thirst. ${ }^{18,20,21}$ Hormones are a major factor in patients with cold RA compared with patients with hot $\mathrm{RA},{ }^{18}$ with higher rates of fat and protein mobilization and lower levels of acylcarnitine, indicating reduced muscle mass and more pronounced muscle rupture. Hypothalamic-pituitaryadrenal axis function decline in cold RA patients is associated with a reduced stress response, which leads to a lack of response to stress factors, leading to the persistence of autoimmune and vocal inflammatory processes. CRP I activity in the cold RA group decreases, and carnitine homeostasis changes, which may be one reason for increases in body fatigue levels. Carnitine and acylcarnitine supplementation may be beneficial for cold RA patients, but less for hot RA patients. ${ }^{22}$
The differences in the biological backgrounds (genes, proteins, and metabolites) of patients with RA cold and hot symptoms have been reported in the study system, ${ }^{23,24}$ and these results broaden the prospect of personalized RA treatment.

\section{Animal model of RA related research}

RA is deemed to be an autoimmune disease. The pathology of RA is uncertain. The establishment and application of animal models is an effective method for the study of RA, especially through the use of animal models similar to those of human beings. Animal models are useful for understanding the pathogenesis of RA. Appropriate animal models should be selected according to experiments, because they have unique characteristics. Various methods have been applied to induce arthritis in animal experimental models, providing essential insight into the pathogenesis of human RA. In addition to rodent (self-onset and external factor-induced) models, there are also related inflammatory cells, such as inflammatory synovial cells, involved in the animal models used in arthritis-related research.

\subsection{Arthritis models in genetically engineered animals}

A series of genetically modified and transgenic mice can be used to analyze complex pathological events (Table S1†). In this review, we briefly introduce the classical TNF transgenic mouse model. In 1991, transgenic mice developed by J. Keffer et al. overexpressed human TNF- $\alpha .{ }^{25}$ Mice developed chronic inflammatory polyarthritis and human TNF anti-monoclonal antibodies were utilized to completely prevent the disease. Since then, multi-line TNF transgenic mice have highlighted the importance of TNF- $\alpha$ with respect to the cytokine levels of RA, and demonstrated the effectiveness of anti-TNF- $\alpha$ in the human body. TNF transgenic mice provide a powerful tool to study RA.

\subsection{Adjuvant-induced arthritis}

Adjuvant arthritis is the earliest animal model of induced arthritis. ${ }^{26}$ An inactivated mycobacteria suspension in oily emulsion was injected subcutaneously into the tail root of experimental animals, ${ }^{27}$ and this gradually developed into arthritis and caused severe pain in about two weeks. The pathological features were that lymphocytes infiltrated into the synovium, the foot claw volume increased and bone damage was caused. ${ }^{27}$ This model was mainly used to test the analgesic effects of nonsteroidal anti-inflammatory drugs (NSAIDs) as early as the 1990s, but it has been reported that many drugs for the treatment of RA cannot significantly improve the pathological state of RA, so their application is slightly limited. ${ }^{28}$

\subsection{Collagen-induced arthritis}

Collagen-induced arthritis (CIA) is a commonly used animal model. Type II collagen is emulsified in incomplete Freund's adjuvant to induce CIA in experimental animals. This arthritis model was first developed in rats. ${ }^{29}$ Later, mostly mice were 
used. ${ }^{30}$ CIA has many similarities with human RA. The CIA model disrupts tolerance and produces the twin features of autoantibodies acting against the subject itself and collagen, making CIA a gold standard in vivo model for RA studies. ${ }^{31}$ The study of the CIA model has established the important roles of $\mathrm{T}$ cells and $\mathrm{B}$ cells in the development of arthritis. ${ }^{32}$ The CIA model has turned out to be useful for developing concepts that can subsequently be extended to humans and used to validate new therapeutic goals.

\subsection{TCM theory-integrated diseases and syndrome models}

$\mathrm{RA}$ is a Bi syndrome in TCM terms; the preparation of a suitable experimental model involves the effects of increasing physical environmental factors in the induced RA model, such as allowing rats injected with the collagen to swim in ice water. ${ }^{33}$ The preparation method of this model is to simulate the influence of an external wind-cold environment on the body in TCM.

\subsection{Other types of arthritis}

In addition to classical adjuvant-induced arthritis and widely used CIA experimental models, there are arthritis animal models induced by antibodies and chemical reagents, such as arthritis induced by collagen antibodies and zymosan-induced arthritis. $^{34,35}$ Rodent models are the only examples here, and some cell models have been widely used in studies of targeting mechanisms, such as biological signaling pathways. In a word, the models studied in the direction of arthritis can mainly be divided into spontaneous arthritis and induced arthritis. Although they are different subtypes, they both have certain common characteristics, ${ }^{36}$ such as the local inflammation of large and small joints, and, in addition, the role of cytokines in the process of arthritis, synovitis caused by these progressive pathological factors, pannus and cartilage erosion, and other RA phenomena.

\section{Effects of CHMs and their extracts on RA}

A flexible combination of CHMs and their natural products guided by TCM theory can play a preventive and therapeutic role for patients with different syndromes. ${ }^{37}$ The prescription of CHMs has been extensively used in clinical practice in China and has gradually resulted in standards and systems with the approval of the government. With the development of TCM modernization, the effectiveness of TCM is gradually being clarified. For example, through using chemi-omics to discuss the efficacy of substances, ${ }^{38}$ serum pharmaceutical chemistry technology using CHMs can explain what kind of relationship exists between the active components and the metabolism in blood containing CHM complex components after entering the human body. Many studies have found that the main active components of CHMs are alkaloids, flavonoids, triterpenes, quinones, volatile oils, steroids and polysaccharides, which have also been studied and found to play a certain pharmacological role in the biological mechanisms of many diseases. ${ }^{39-41}$
Similarly, this is also reflected in research into $\mathrm{RA},{ }^{\mathbf{4 2 , 4 3}}$ where classical prescriptions are the Wutou decoction, ${ }^{\mathbf{4 4}}$ Guizhi Shaoyao Zhimu decoction, ${ }^{\mathbf{4 5}}$ Simiao pill, ${ }^{\mathbf{4 6}}$ Juanbi decoction, ${ }^{\mathbf{4 7}}$ etc. We have systematically collated the CHMs and their compounds that have an effect on rheumatoid arthritis (as shown in Fig. 1, Tables S2 and S3†).

\section{The potential mechanisms of TCM to antagonize RA}

As an inflammatory disease with abnormal immune function, RA has a gradual process of pathogenesis and, ultimately, pathological phenomena such as articular cartilage erosion and bone damage are due to synovial inflammation. Of course, there are various microscopic factors involved in these unwanted phenomena. Some inflammatory factors and adhesion molecules, and many inflammatory-promoting signaling pathways have caught the attention of researchers. ${ }^{.5,51}$ The focus on TCM is gradually increasing in RA-related research, mainly due to studies of the effects of CHMs and their extracts on RA synovitis, vasospasm, bone damage, immune cell abnormalities and other reactions (Fig. 2). The expression of TCM mechanisms acting on RA will be briefly discussed next.

\subsection{Application of omics technology to CHM antagonistic RA}

The development of analytical techniques and methods has accelerated the maturity of omics. Many of them, including genomics, proteomics, metabonomics and transcriptome, have made indelible contributions to the field of life science, because they reflect the function and metabolism of human tissues and organs through the overall analysis, and provide new ideas for exploring the pathogenesis of human diseases. ${ }^{52}$ Omics has also been widely used in many studies of RA. The discovery of related genes, proteins and biomarkers at metabolic level increases the height of RA related research. ${ }^{53}$

\subsection{Inhibition of synovial inflammation and vascular proliferation}

Synovial tissue is mainly composed of fibroblast-like synovial cells. Fibroblast-like synovial cells can secrete inflammatory cytokines, chemokines and matrix protein degradation enzymes, ${ }^{54}$ and these biological factors may cause synovial cells to enter a pathological state, such as how synovial tissue cell signal conduction abnormalities can result in its proliferation and apoptosis imbalance. ${ }^{55}$ Konisti S. and Meng both reported that the onset of RA could be facilitated via hypoxia, ${ }^{56}$ because hypoxia inducible transcription factor- $1 \alpha$ (HIF-1 $\alpha)$ may activate and increase the expression of viable genes involved in signal transduction and energy metabolism in RA fibroblasts in low oxygen environments. ${ }^{57}$ In addition, there have been studies suggesting that toll-like receptors (TLRs) are adaptive immune response modulators; ${ }^{58}$ TLRs in patients with excessive RA immune system activation can cause monocytes to produce TNF- $\alpha$ and other chemokines. ${ }^{59}$ TNF- $\alpha$ is a kind of inflammatory mediator with many biological effects, which may stimulate the proliferation of synovial fibroblasts. ${ }^{6}$ Researchers have 

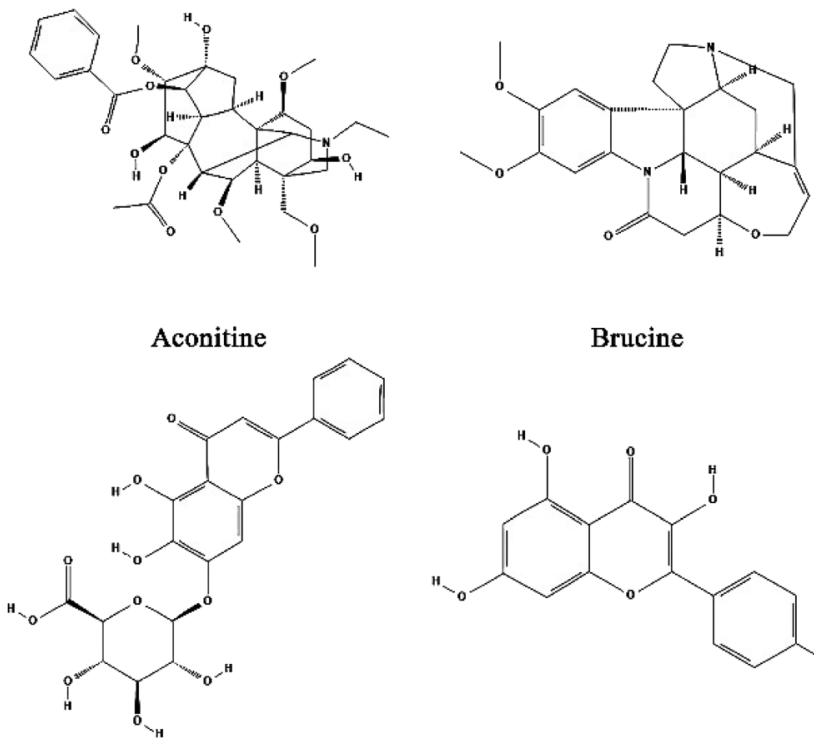

Baicalin

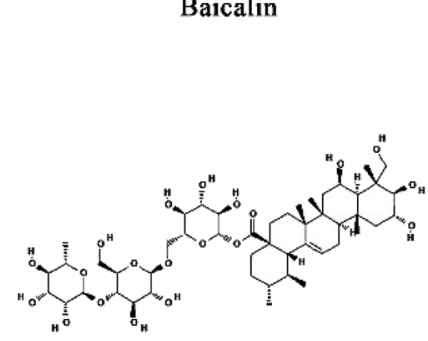

Asiaticoside A

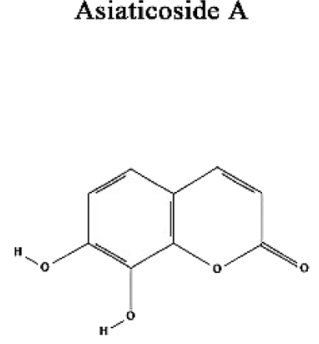

Daphnetin
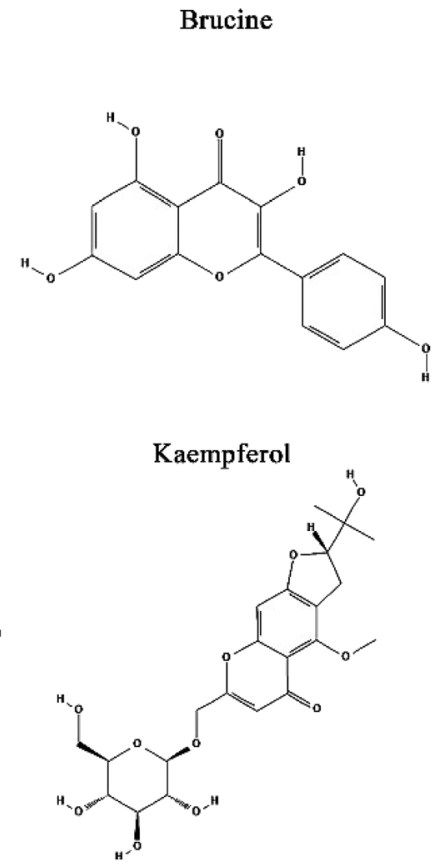

Prim-O-glucosylcimifugin

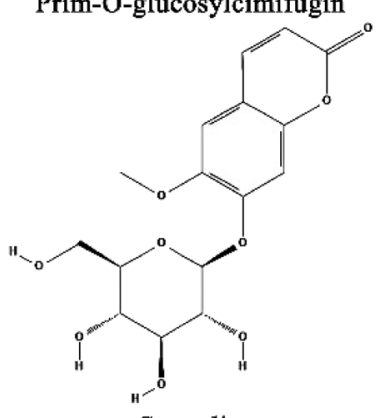

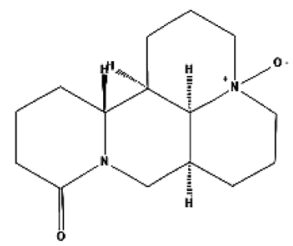

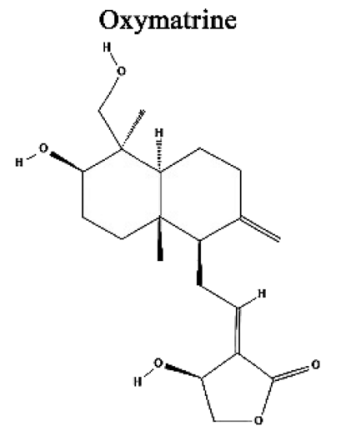

Andrographolide<smiles>COc1ccc2c(c1OC)C1(CCN2C)CC(C)C(OC)C[C@H]1N</smiles>

Sinomenine

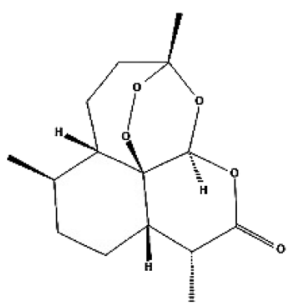

Artemisinin

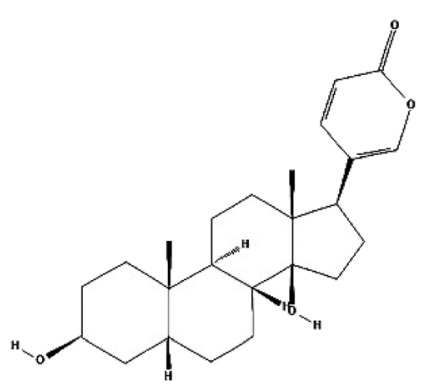

Bufalin

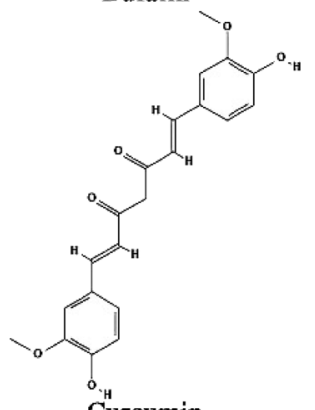

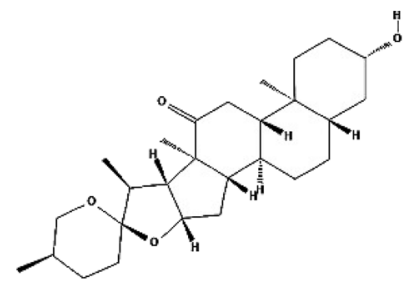

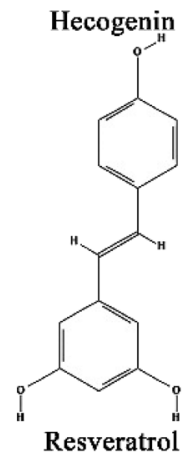

Fig. 1 Common anti-RA compounds.

concluded that herbal extracts of paeonol could inhibit the expression of the direct target FOXO3 of miR-155, the most important of which is the inhibition of TNF induction. ${ }^{61} \mathrm{IL}-6$ can induce hepatocytes to synthesize diverse acute phase reaction proteins, promote B cell synthesis, and produce immunoglobulin and rheumatoid factors. ${ }^{62}$ It is worth noting that Yang et al. ${ }^{63}$ used RT-qPCR and Western blotting to observe the effect of the CHM Aconitum leucostomum Worosch on human fibroblast-like synovial cell rheumatoid arthritis (HFLS-RA) cells. The results showed that the apoptosis rate of the Aconitum leucostomum Worosch group was significantly higher than that of the control group $(P<0.05)$, and the expression levels of HIF-1 $\alpha$, TLR4 mRNA and proteins were substantially down- regulated $(P<0.05)$ in the cell culture supernatant from HFLSRA cells $(P<0.05)$. The levels of TNF- $\alpha$ and IL-6 were significantly decreased (all $P<0.05$ ). From these results we can see that A. leucostomum Worosch crude drug processed products and monomers could have an anti-rheumatic effect on HFLS-RA cells, inhibit cell proliferation and enhance cell apoptosis. These effects can be attributed to the downregulation of HIF-1 $\alpha$ and TLRs expression and the decrease in pro-inflammatory cytokines. There is also much evidence showing a close relationship between fibroblast activation and extracellular matrix (ECM) component recombination in RA synovial tissue fibrosis, while the conversion of transforming growth factor- $\beta$ (TGF- $\beta$ ) plays a key role in the fibrosis reaction. ${ }^{64}$ Chen suggests that 
ATX-LPA MAPK JAK2/STAT3 TGF- $\beta$ IL-6 IL-17 PGE-2

TNF- $\alpha$ IFN- $\gamma$ IL-2 IL-1 $\beta$ VEGF TLR4 MyD88 NF-кB

Th1 Th17 Th2 Treg RANKL-RANK-OPG MMP-1 MMP3

Phenylalanine metabolism Tyrosine and tryptophan biosynthesis

Taurine and hypotaurine metabolism TCA cycle

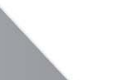

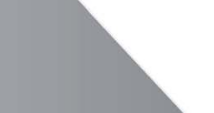

\section{.}

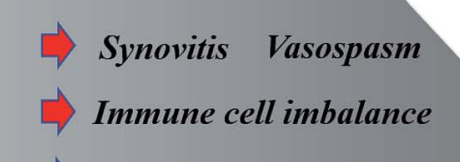

Bone damage

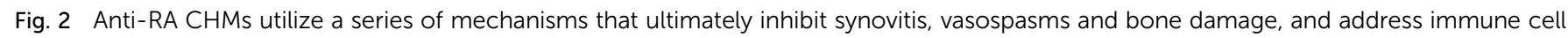
imbalances.

TGF- $\beta$ is a secreted protein that regulates cell differentiation, tissue proliferation and fibrogenesis. ${ }^{65}$ TGF- $\beta 1$ activates myofibroblasts through two signal transduction pathways when inflammation occurs. This may lead to the abnormal composition of the synovial ECM and then synovial fibrosis, ${ }^{66}$ in which TGF- $\beta 1$ is accumulated in the ECM. The activation and proliferation of myofibroblasts and the high expression of $\alpha$-smooth muscle actin ( $\alpha$-SMA) may be the obvious pathological responses of myofibroblast fibrosis. ${ }^{67}$ It is worth mentioning that succinic acid participates in the anaerobic respiration of all cells as an intermediate in the citric acid cycle under hypoxic conditions, ${ }^{68}$ while Tannahill G. M. and other researchers have shown that succinic acid acts on HIF-1 $\alpha$ in macrophages and increases the secretion of the inflammatory mediator IL-1 $\beta,{ }^{69}$ so succinic acid may be fibroblast-forming. The key point is inflammation in a hypoxic environment. In the synovial cells of $\mathrm{RA}$ rats, due to the reversal of succinic dehydrogenase (SDH) activation and a dependence on HIF- $1 \alpha$ induced NACHT, LRR and PYD domain-containing protein 3 (NLRP3) inflammatory body activation, hypoxic TGF- $\beta 1$ induces increased succinate accumulation and responds to NLRP3 inflammatory factor activation, releasing IL- $1 \beta$ to further increase TGF- $\beta 1$ induction, indicating a vicious cycle between inflammation and fibrosis in the activation of muscle fibroblasts. Clematichinenoside AR (Car) is a TCM used in the treatment of arthritis obtained by separating three saponins from the roots of Clematis mandshurica Rupr. C-ar has anti-inflammatory and immune inhibition effects; $\mathrm{Li}$ and other researchers found that $\mathrm{C}$-ar can inhibit the activity of succinic dehydrogenase, thus breaking the vicious cycle between NLRP3 inflammatory mediators, succinic acid and TGF- $\beta 1$, resulting in the inhibition of inflammation and fibrosis of the fibroblasts. ${ }^{49}$

Several studies have shown that the formation of RA is closely related to the proliferation of blood vessels; ${ }^{70}$ neovascularization can promote inflammatory cells into the bones and joints and cause synovial hyperplasia and bone destruction. ${ }^{71}$ VEGF is a growth factor secreted by macrophages and synovial tissue fibroblasts in RA, ${ }^{72}$ which is a key regulatory factor for angiogenesis. VEGF can promote the proliferation and migration of endothelial cells to support the emergence of new blood vessels. The accumulation of HIF- $1 \alpha$ in the cytoplasm and the positive feedback regulation of the expression and secretion of VEGF and HIF- $1 \alpha$, and the VEGF pathway in macrophages and fibroblasts of RA synovial tissue can trigger angiogenesis during hypoxia. ${ }^{73}$ Paclitaxel (PTX) is a natural diterpenoid alkaloid compound extracted from dry endothelium of Taxus brevifolia. It mainly works against the structures of cells. ${ }^{74}$ In addition, it has been proved that PTX can regulate immune function in response to cancer. It can inhibit the expression of $\mathrm{VEGF}^{75}$ and HIF- $1 \alpha{ }^{76}$ and has anti-angiogenesis effects. Results have shown that PTX could significantly decrease the expression of VEGF and HIF-1 $\alpha$ in the synovial tissue and peripheral serum of CIA mice, while VEGF and HIF$1 \alpha$ might be potential therapeutic targets for PTX to inhibit the microvascular progression of RA. ${ }^{77}$ This view was further confirmed by researchers who found that succinate activates VEGF expression in cells, through induction dependent on HIF$1 \alpha .^{78}$ Because HIF- $1 \alpha$ is a transcription factor related to angiogenesis, the resulting HIF- $1 \alpha$ is regulated by prolyl hydroxylase domain enzymes, with the hydroxylation of HIF- $1 \alpha$ promoting 
its degradation. ${ }^{79}$ On the one hand, dimethyl succinate can damage the hydroxylation of HIF- $1 \alpha$ and increase the accumulation of HIF- $1 \alpha$ in endothelial cells, promoting the transcription of HIF- $1 \alpha$ into the nucleus. On the other hand, it is found that HIF- $1 \alpha$ is necessary for succinic acid to induce the VEGF gene promoter. This also demonstrates that succinic acid stimulates the production of VEGF through the transcriptional regulation of HIF-1 $\alpha$. Succinic acid activates the G-proteincoupled receptor 91 in vitro and disturbs the energy metabolism, with increased inflammation and angiogenesis in the synovium. It has also been shown that the excessive accumulation of succinic acid can not only cause synovitis but also induce vascular proliferation. The classic TCM aconitum decoction (WTD) is widely utilized in the treatment of RA; the studies of He et al. have shown that WTD doses of 0.95, 1.9 and $3.8 \mathrm{~g} \mathrm{~kg}^{-1}$ can reduce inflammatory synovial tissue in the immature blood vessels of CIA rats. ${ }^{2}$ In vitro and in vivo experiments have shown the mechanism of its role; WTD blocked the self-phosphorylation of VEGF165-induced VEGFR2, and therefore lowered the VEGF165 activation of AKT, ERK1/2, JNK and the p38 signal transduction pathway, significantly reducing the expression of angiogenesis activators, including vascular endothelial growth factor (VEGF), IL-1 $\beta$, IL-17, transforming growth factor- $\beta$, angiogenic I (Ang I) and Ang II, thus resisting the formation of vasospasms. Huang and other researchers have also studied the anti-angiogenesis mechanism of Croton crassifolius, a medicinal plant in the Morinda family. It was found that the supercritical fluid extract involved in the signal pathway $\mathrm{Kdr}$, which is closely related to VEGF, played an important role in the formation of vascular endothelial cells, ${ }^{80}$ which is of great significance for the clinical treatment of RA. In $\mathrm{RA}, \mathrm{NF}-\kappa \mathrm{B}$ is overexpressed in inflammatory synovium; ${ }^{81}$ this is associated with the transcription activation of numerous genes and mediates immune and inflammatory responses. ${ }^{82}$ It can be reinforced by TNF- $\alpha$, IL-1, PGE and IL-6. Inflammatory cytokines are activated, thus forming a positive regulatory circle, causing the amplification of the local inflammatory response. Guo et al. extracted Caulophyllum robustum Maxim (C. robustum), whose main component was $C$. robustum extraction (CRME), and used lipopolysaccharide (LPS)-stimulated RAW264.7 cells and CIA DBA/1 in mice to study the antiinflammatory effects of CRME. ${ }^{83}$ It was found that CRME can reduce the levels of IL-1, IL-6, TNF- $\alpha$, and PGE2, and inhibit the expression of NF- $\kappa \mathrm{B}$ p 65 , thereby reducing TNF- $\alpha$, the migration of leukocytes to the RA joints, the excessive growth of blood vessels caused by IL-6, and the pain sensitivity of IL-1 and PGE2, finally leading to the inhibition of RA.

\subsection{Inhibition of bone erosion}

Bone remodeling is a process of balancing osteoblasts and osteoclasts. The balance between osteoblasts and osteoclasts can avoid diseases such as RA resulting from excessive bone resorption. ${ }^{84}$ Osteoclasts are polynuclear cells from bone resorption, which are fused by dendritic cell-specific transmembrane protein (DCSTAMP) and other fusion molecules to regulate the mononuclear macrophage lineage of hematopoietic stem cells. ${ }^{85}$ The regulation of bone remodeling is strictly contingent on the activity of the RANKL-RANK-OPG axis. ${ }^{86}$ The receptor activator for the NF- $\kappa \mathrm{B}$ ligand is a fellow of the TNF ligand superfamily, and is produced by osteoblasts, binds to its homologous receptors and expresses in osteoclast progenitor cells, mature osteoclasts and chondrocytes. ${ }^{87}$ Meanwhile, pro-inflammatory cytokines such as TNF- $\alpha$ and IL-6 can induce the expression of RANKL mesenchyme stem cells (MSCs) and promote the formation of osteoclasts. ${ }^{88}$ MSCs can secrete a large amount of the soluble protein OPG, which can inhibit the gene expression of the nuclear factors of activated $\mathrm{T}$ cells, cytoplasmic 1 (NFATc1) and creatine kinase (CK), ${ }^{89}$ thus competitively inhibiting the RANKL locus, so that OPG can indirectly inhibit the formation and activation of osteoclasts..$^{90}$ On the other hand, MSCs can secrete soluble molecules, such as PGE2, to participate in immunosuppression. Zhou and other researchers found that sinomenine, the root extract of Sinomenium acutum, can regulate the expression of PGE2 in MSC, thereby inhibiting the expression of RANKL and osteoclast formation. ${ }^{89}$ Studies have shown that there are many factors involved in osteoclast and osteoblast formation, such as GM-CSF and osteopontin (OPN). ${ }^{91} \mathrm{~A}$ variety of immune cells can produce GM-CSF, which can govern the fusion of osteocytes. ${ }^{92}$ OPN generated by synovial fibroblasts promotes osteoclast activity and inhibits osteoblast activity. Interestingly, Huo-Luo-Xiao-Ling Dan results in an inhibition of bone damage during RA. The protection from bone damage induced by HLXL is mainly through inhibiting the interfering factors of bone cell remodeling (such as NF- $\mathrm{B}$, RANKL, GM-CSF and OPN), adjusting the RANKL/OPG ratio, which is beneficial for the antiosteoclast activity and reducing the number of osteoclasts in the joint bone. It can be concluded that HLXL treatment is directed at the bone. A critical medium level of remodeling has a regulatory effect. ${ }^{86} \mathrm{IL}-1 \beta$ can inhibit the synthesis of proteoglycan and modify the synthesis and metabolism of chondrocytes. By stimulating chondrocytes to induce the MAPKs signaling pathway, the degradation of proteoglycans can be enhanced, resulting in the expression of various inflammatory mediators, including matrix metalloproteinases (MMPs), such as MMP-1, MMP-3 and MMP-13, and aggregating proteoglycan enzymes. ${ }^{93}$ MMPs play a crucial role in the process of RA joint bone injury, in which MMP-1 and MMP-3 can directly change the composition of the ECM as key enzymes in the remodeling and degradation of the ECM. An aqueous solvent extraction of Eucommia ulmoides can reduce their levels, ${ }^{94}$ and similar studies have shown that the compound Fu-Fang-Lu-JiaoShuang can regulate the ratios of tissue inhibitors of metalloproteinase and MMPs to protect from bone injury. ${ }^{95}$ It has further been noted that the binding of RANKL to RANK leads to the synthesis of tumor necrosis factor receptor-associated factor 6 , which activates transcription factors such as JNK and p38. For example, the transcription factors NF- $\mathrm{B}$, activator protein 1 and activated $\mathrm{T}$ cell factor ${ }^{96}$ regulate osteoclast differentiation through upregulating the expression of osteoclast related genes such as TRAP, cathepsin K and DC-STAMP. In the process of osteoclast differentiation, the fusion of preosteoclasts is an important event in the formation of mature polynuclear osteoclasts. The failure of fusion may lead to a serious decrease in bone resorption activity and an increase in bone accumulation. DC-STAMP plays an important role in mononuclear osteoclast fusion. Cells lacking DC- 
STAMP cannot develop into polynuclear osteoclasts, thus inhibiting bone resorption. ${ }^{97}$ It was found that aconitine, the main active component of aconitum, inhibited the activation of NF- $\mathrm{B}$ and NFATc1 and the expression of osteoclast-specific genes and DCSTAMP in a dose-dependent manner, inhibiting the differentiation of osteoclasts induced by RANKL in RAW264.7 cells. ${ }^{98}$ It has been reported that asporin (ASPN) is a small type 1 leucine polysaccharide (SLRP), with high levels of SLRP, which can reduce the expression of TGF $\beta 1$ in the synovial tissue of joints, and lead to collagen fiber metabolism disorder. ${ }^{99}$ The leukemia inhibitory factor receptor (LIFR) is a subunit of the LIF receptor. LIF produced by muscle cells is a multifunctional soluble IL-6 family cytokine, which affects skeletal muscle regeneration via stimulating the production of pro-inflammatory factors in joints. ${ }^{\mathbf{1 0 0}}$ The overexpression of LIFR activates ligands and common signal transduction mediators, aggravating the inflammation of chondrocytes in synovial tissue. ${ }^{\mathbf{1 0 1}}$ It has been found that paeoniflorin can reduce the expression of ASPN and LIFR associated with RA, ${ }^{102}$ and then inhibit the inflammation of chondrocytes.

\subsection{Inhibition of immune cell imbalances}

The accumulation of inflammatory cells, the proliferation of synovial tissue and the destruction of articular bone in patients with RA are related to imbalances in the immune network, including $\mathrm{T}$ and $\mathrm{B}$ cells and macrophages. The abnormal differentiation of inflammatory cells, such as dendritic cells and natural killer cells, can lead to synovial and vascular proliferation in patients with $\mathrm{RA},{ }^{\mathbf{1 0 3 , 1 0 4}}$ and cartilage destruction and bone erosion in the sublayer. ${ }^{105}$ Of these, $\mathrm{T}$ cell subsets have attracted the attention of numerous researchers, while $\mathrm{T}$ helper 1 (Th1), Th2, Th17, and regulatory T cells (Treg) play a relatively wide role in RA. Treg cells have the function of inhibiting autologous reactive lymphocytes. ${ }^{48}$ However, the aberrant differentiation of Th1 increases the secretion of IFN- $\gamma$, while Th2 cells produce the cytokines IL-4, IL-5 and IL-13. ${ }^{106}$ IFN- $\gamma$ binds to the corresponding receptors and activates STAT1, which then promotes the expression of T-bet-encoded Tbx21 genes. T-bet also increases INF- $\gamma$. Importantly, T-bet also acts on IL-12 receptors and further enhances IFN- $\gamma$ production, producing a positive cycle. T-bet inhibits the expression of GATA-3 genes to prevent the differentiation of Th2.$^{\mathbf{1 0 7}}$ The IL-4 receptor signal promotes the intense expression of IL-4 and GATA- 3 genes and differentiates Th2 cells with resistance. GATA- 3 can also inhibit the expression of $\beta 2$ chains of the IL-12 receptor and limit the differentiation of $\mathrm{Th} 1,{ }^{108}$ which leads to an imbalance of Th1-Th2 cells. The expression of the IL-17 receptor and the nuclear receptor retinoic acid-related orphan receptor $\gamma$ receptor gene in Th17 cells can increase the secretion of IL-17 by Th17 cells, ${ }^{109}$ which leads to synovial inflammation, leading to cartilage and bone destruction. These phenomena may be closely linked to the loss of control of effector T cells by Treg. B. Nie et al. found that the TCM Xianfanghuomingyin can regulate the proliferation and differentiation of $\mathrm{T}$ lymphocytes in collagen induced mice, having a positive effect on RA. ${ }^{\mathbf{1 0 5}}$ The mechanism involves the regulation of cytokines and inflammatory factor IFN- $\gamma$ associated with $\mathrm{T}$ cell differentiation, T-bet,
IL-4 and GATA-3 levels, in turn inhibiting the proliferation and activation of functional CD3 + CD4 + T cells, and conversely promoting the transformation of T cells into CD4 + CD25 + Treg cells with regulatory functions. In addition, with XFHM it is possible to inhibit the differentiation of Th1 and Th17 cells and increase the differentiation of Th2 cells. There is a suggestion that celastrol can regulate the $\mathrm{T}$ cell balance. ${ }^{\mathbf{1 1 0}}$ Researchers used an AIA rat model to observe the effects of tripterine on regulating the number of Th17/Treg cells. It can inhibit the production of Th17 differentiation cytokines and chemokines (CCL 3 CCL5), block the activation of STAT 3 and decrease the amount of Th17. It can also promote the differentiation of Treg cells and slow down the pathological inflammatory reaction. Related studies have shown that Janus tyrosine kinase (JAK) signal transduction and transcriptional activator (STAT) signaling pathways can mediate the differentiation of $\mathrm{T}$ cells at inflammatory sites, ${ }^{\mathbf{1 1 1}}$ and activated CD4 $\mathrm{T}$ cells differentiate Th17 cells through a STAT3 pathway. ${ }^{112}$ The inhibition of the activation of the JAK2/STAT3 signaling pathway and the differentiation of Th17 cells may be an approach for the treatment of RA. Interestingly, Xin et al. observed the mechanism of Wenjinghuoluo (WJHL) in regulating Th17 cells. The results showed that WJHL reached its goal by inhibiting the JAK2-STAT3 signal transduction pathway, finally inhibiting synovial inflammation and cartilage injury. ${ }^{113}$ In conclusion, the operational balancing of the number of $\mathrm{T}$ cell subsets may become a novel method for the treatment of autoimmune diseases such as rheumatoid arthritis, making it worthy of further exploration.

\section{Discussion and conclusions}

At present, common cognition of the pathogenesis of RA focuses on the immune abnormalities caused by the innate and external environments, which will attract many inflammatory mediators to focus on the large joints, leading to synovitis and bone damage. ${ }^{\mathbf{1 1 4 1 1 5}}$ TCM has its own theories and strategies relating to the function of the disease, from the dialectical type to symptomatic treatment; this is conducted under the guidance of clinical specifications. TCM uses CHMs and their natural products to affect RA, and it is undeniable that the above-mentioned CHM compounds and their herbal extracts have an antagonistic effect on RA patients and experimental animal models, which can be described through the inhibition of synovitis, vascular hyperplasia, bone disorders and immune cells being out of balance. It is well known that CHMs and their natural products possess many biological activities; antiinflammatory and antioxidative stress effects are the principal contributors to their pharmacological action. ${ }^{116-118}$ However, it is worth mentioning that researchers have used omics techniques and methods to study the mechanism of RA in transgenic and induced arthritis animal models, and many of the results are satisfactory; however, these phenomena do not appear to be specific. In other words, it is found that the functions or inhibitory effects of CHMs and their natural products on these targets and pathways are also found for other diseases, not only RA, such as in the case of NF- $\kappa$ B, TNF- $\alpha$ and members of the interleukin family. ${ }^{\mathbf{1 1 6 , 1 1 9}}$ We urgently need to find a new, 
highly specific underlying story for RA, of course relating to current research methods and results. To sum up, the study of the mechanisms of TCM and CHMs on RA in terms of systemic biology has found that they can improve the pathological states of RA patients and experimental animal models, as it is possible to regulate the production of cytokines and chemicals, reducing the proliferation of pathogenic genes and inducing apoptosis, and modulating the expression of inflammatory mediators to alleviate oxidative stress. Finally, the inflammatory responses in joints are alleviated, the excessive proliferation of the synovial membrane and vascular wall cells is alleviated, and the imbalance of osteoclasts is regulated to avoid bone damage.

\section{Conflicts of interest}

There are no conflicts to declare.

\section{Acknowledgements}

This work was supported by grants from the National Key Research and Development Program of China (2018YFC1706103), the Key Program of the Natural Science Foundation of State (Grant No. 81830110, 81430093, 81373930, 81673586, 81703685, 81302905, 81503386), the National Key Subject of Drug Innovation (Grant No. 2015ZX09101043-005, 2015ZX09101043-011), the TCM State Administration Subject of Public Welfare (Grant No. 2015468004), the Major Projects of Application Technology Research and Development Plan in Heilongjiang Province (GX16C003), the Young Talent Lift Engineering Project of China Association of Traditional Chinese Medicine (QNRC2-B06), the Foundation of Heilongjiang University of Chinese Medicine (2018jc01, 2018bs02, 201809, QC2018117), the University Nursing Program for Young Scholars with Creative Talents in Heilongjiang Province (UNPYSCT-2015118, UNPYSCT-2016213, UNPYSCT-2016212), the Application Technology and Development of Youth Talents Project in Harbin (2014RFQXJ116), the Chinese Postdoctoral Science Foundation (2017M621319b), Natural Science Foundation of Heilongjiang Province (YQ2019H030, LH2019H056, H2016056), and the Returned Oversea Scholars Program of Heilongjiang Province (2017QD0025).

\section{References}

1 F. Angelotti, A. Parma, G. Cafaro, et al. One year in review 2017: pathogenesis of rheumatoid arthritis, Clin. Exp. Rheumatol., 2017, 35(3), 368.

2 L. He, C. Liu, C. Sun, et al., Wu-Tou Decoction Inhibits Angiogenesis in Experimental Arthritis by Targeting VEGFR2 Signaling Pathway, Rejuvenation Res., 2018, 21(5), 442-455.

3 J.-Y. Wang, X.-J. Chen, L. Zhang, et al., Comparative Studies of Different Extracts from Eucommia ulmoides Oliv. against Rheumatoid Arthritis in CIA Rats, Evid. Based Complement. Altern. Med., 2018, 7379893.

4 R. Puchner, R. Hochreiter, H. Pieringer, et al., Improving patient flow of people with rheumatoid arthritis has the potential to simultaneously improve health outcomes and reduce direct costs, BMC Musculoskeletal Disord., 2017, 18(1), 7.

5 F. Wolfe, D. M. Mitchell, J. T. Sibley, et al.The mortality of rheumatoid arthritis, Arthritis Rheum., 2014, 37(4), 481-494.

6 M. H. Ma, A. P. Cope and D. L. J. I. J. O. C. R. Scott, Safety of combination therapies in early rheumatoid arthritis: a systematic comparison between antirheumatic drugs and TNF inhibitors with methotrexate, Int. J. Clin. Rheumatol., 2010, 5(5), 547-554.

7 D. Tecer, M. Sezgin, A. Kanık, et al.Can mean platelet volume and red blood cell distribution width show disease activity in rheumatoid arthritis?, Biomarkers Med., 2016, 10(9), 967-974.

8 T. Vos, C. Allen, M. Arora, et al., Global, regional, and national incidence, prevalence, and years lived with disability for 310 diseases and injuries, 1990-2015: a systematic analysis for the Global Burden of Disease Study 2015, Lancet, 2016, 388(10053), 1545-1602.

9 J. S. Smolen, D. Aletaha and I. B. Mcinnes, Rheumatoid arthritis, Lancet, 2016, 388(10055), 2023-2038.

10 A. Alex, C. Serena and M. F. J. Bmj, Diagnosis and management of rheumatoid arthritis in adults: summary of updated NICE guidance, BMJ, 2018, 362, k3015-k3019.

11 L. M. Mota, B. A. Cruz, C. V. Brenol, et al. Guidelines for the drug treatment of rheumatoid arthritis, Rev. Bras. Reumatol., 2013, 53(2), 158-183.

12 X. N. Li, A. Zhang, M. Wang, et al., Screening the active compounds of Phellodendri amurensis cortex for treating prostate cancer by high-throughput chinmedomics, Sci. Rep., 2017, 7, 46234.

13 X. Li, A. Zhang, H. Sun, et al., Metabolic characterization and pathway analysis of berberine protects against prostate cancer, Oncotarget, 2017, 8, 65022-65041.

14 H. Sun, A. H. Zhang, Q. Song, et al., Functional metabolomics discover pentose and glucuronate interconversion pathways as promising targets for Yang Huang syndrome treatment with Yinchenhao Tang, RSC Adv., 2018, 8, 36831-36839.

15 J. Fang, N. Zheng, Y. Wang, et al., Understanding Acupuncture based on Zheng Classification from System Perspective\%, J. Evidence-Based Complementary Altern. Med., 2013, 2013, 10.

16 A. Zhang, G. Yan, Y. Han and X. Wang, Metabolomics approaches and applications in prostate cancer research, Appl. Biochem. Biotechnol., 2014, 174, 6-12.

17 M. Wang, G. Chen, C. Lu, et al., Rheumatoid arthritis with deficiency pattern in traditional chinese medicine shows correlation with cold and hot patterns in gene expression profiles, Evid. Based Complement. Altern. Med., 2013, 2013, 248650.

18 A. Zhang, H. Sun, G. Yan, et al., Metabolomics in diagnosis and biomarker discovery of colorectal cancer, Cancer Lett., 2014, 345, 17-20.

19 X. J. Wang, J. L. Ren, A. H. Zhang, et al., Novel applications of mass spectrometry-based metabolomics in herbal medicines and its active ingredients: current evidence, Mass Spectrom. Rev., 2019, 9999, 1-23. 
20 A. Zhang, H. Sun, Y. Yuan, et al., An in vivo analysis of the therapeutic and synergistic properties of Chinese medicinal formula Yin-Chen-Hao-Tang based on its active constituents, Fitoterapia, 2011, 82, 1160-1168.

21 C. Lu, X. Niu, C. Xiao, et al., Network-based gene expression biomarkers for cold and heat patterns of rheumatoid arthritis in traditional chinese medicine, Evid. Based Complement. Altern. Med., 2012, 2012, 203043.

22 H. Wang, G. Yan, A. Zhang, et al., Rapid discovery and global characterization of chemical constituents and rats metabolites of Phellodendri amurensis cortex by ultraperformance liquid chromatography-electrospray ionization/quadrupole-time-of-flight mass spectrometry coupled with pattern recognition approach, Analyst, 2013, 138, 3303-3312.

23 A. Zhang, H. Sun, X. Wu, et al., Urine metabolomics, Clin. Chim. Acta, 2012, 414, 65-69.

24 H. Guo, X. Niu, Y. Gu, et al., Differential Amino Acid, Carbohydrate and Lipid Metabolism Perpetuations Involved in a Subtype of Rheumatoid Arthritis with Chinese Medicine Cold Pattern, Int. J. Mol. Sci., 2016, 17(10), 1757-1769.

25 J. Keffer, L. Probert, H. Cazlaris, S. Georgopoulos, E. Kaslaris, D. Kioussis and G. Kollias, Transgenic mice expressing human tumour necrosis factor: a predictive genetic model of arthritis, EMBO J., 1991, 10(13), 40254031.

26 Y. Zhang, P. Liu, Y. Li, et al., Exploration of metabolite signatures using high-throughput mass spectrometry coupled with multivariate data analysis, $R S C A d v ., 2017,7$, 6780-6787.

27 D. M. Meyer, M. I. Jesson, X. Li, et al., Anti-inflammatory activity and neutrophil reductions mediated by the JAK1/ JAK3 inhibitor, CP-690,550, in rat adjuvant-induced arthritis, J. Inflammation, 2010, 7, 41.

28 Y. Li, S. Qiu, A. H. Zhang, et al., High-throughput metabolomics to identify metabolites to serve as diagnostic biomarkers of prostate cancer, Anal. Methods, 2016, 8, 3284-3290.

29 Q. Liang, C. Wang, B. Li, et al., Metabolomics of alcoholic liver disease: a clinical discovery study, $R S C A d v$., 2015, 5, 80381-80387.

30 J. S. Courtenay, M. J. Dallman, A. D. Dayan, et al.Immunisation against heterologous type II collagen induces arthritis in mice, Nature, 1980, 283(5748), 666-668.

31 D. L. Asquith, A. M. Miller, I. B. Mcinnes, et al., Animal models of rheumatoid arthritis, Eur. J. Immunol., 2009, 39(8), 2040-2044.

32 A. Zhang, H. Sun, X. Wang, et al., Recent highlights of metabolomics for traditional Chinese medicine, Pharmazie, 2012, 67, 667-675.

33 A. Zhang, H. Sun, P. Wang, et al., Modern analytical techniques in metabolomics analysis, Analyst, 2012, 137, 293-300.

34 E. C. Keystone, H. U. Schorlemmer, C. Pope, et al.Zymosaninduced arthritis: a model of chronic proliferative arthritis following activation of the alternative pathway of complement, Arthritis Rheum., 1977, 20(7), 1396-1401.

35 H. Xiong, A. H. Zhang, Q. Q. Zhao, et al., Discovery and screening quality-marker ingredients of Panax quinquefolius using chinmedomics approach, Phytomedicine, 2019, S0944, 30097-2.

36 K. D. Moudgil, P. Kim and E. Brahn, Advances in rheumatoid arthritis animal models, Curr. Rheumatol. Rep., 2011, 13(5), 456-463.

37 H. Sun, A. Zhang, L. Yang, et al., High-throughput chinmedomics strategy for discovering the qualitymarkers and potential targets for Yinchenhao decoction, Phytomedicine, 2019, 54, 328-338.

38 A. H. Zhang, H. Sun, G.-L. Yan, et al., Chinmedomics: A Powerful Approach Integrating Metabolomics with Serum Pharmacochemistry to Evaluate the Efficacy of Traditional Chinese Medicine, Engineering, 2018, 5, 60-68.

39 H. Wang, H. Sun, A. Zhang, et al., Rapid identification and comparative analysis of the chemical constituents and metabolites of Phellodendri amurensis cortex and Zhibai dihuang pill by ultra-performance liquid chromatography with quadrupole TOF-MS, J. Sep. Sci., 2013, 36, 874-3882.

40 X. Wang, H. Lv, A. Zhang, et al., Metabolite profiling and pathway analysis of acute hepatitis rats by UPLC-ESI MS combined with pattern recognition methods, Liver Int., 2014, 34, 759-770.

41 A. Zhang, S. Qiu, H. Sun, et al., Scoparone affects lipid metabolism in primary hepatocytes using lipidomics, Sci. Rep., 2016, 6, 28031.

$42 \mathrm{Z} . \mathrm{Wu}, \mathrm{H} . \mathrm{Wu}, \mathrm{C}$. Li, et al., Daphnetin attenuates LPSinduced osteolysis and RANKL mediated osteoclastogenesis through suppression of ERK and NFATc1 pathways, J. Cell. Physiol., 2019, 234(10), 1781217823.

43 X. Liu, A. Zhang, H. Fang, et al., Serum metabolomics strategy for understanding the therapeutic effects of YinChen-Hao-Tang against Yanghuang syndrome, RSC Adv., 2018, 8, 7403-7413.

44 A. Zhang, H. Sun, X. Wang, et al., Mass spectrometry-driven drug discovery for development of herbal medicine, Mass Spectrom. Rev., 2016, 37, 307-320.

45 X. Wang, Q. Wang, A. Zhang, et al., Metabolomics study of intervention effects of Wen-Xin-formula using ultra highperformance liquid chromatography/mass spectrometry coupled with pattern recognition approach, J. Pharm. Biomed. Anal., 2013, 74, 22-30.

46 P. Shen, S. Tu, H. Wang, et al., Simiao Pill Attenuates Collagen-Induced Arthritis in Rats through Suppressing the ATX-LPA and MAPK Signalling Pathways, Evid. Based Complement. Altern. Med., 2019, 2019, 7498527.

47 Q. Wang, Y. R. Wang, Q. Y. Jia, et al., The efficacy of the traditional Chinese medicine Juanbi pill combined with methotrexate in active rheumatoid arthritis: study protocol for a randomized controlled trial, Trials, 2018, 19(1), 188.

48 A. Ma, Y. Yang, Q. Wang, et al., Antiinflammatory effects of oxymatrine on rheumatoid arthritis in rats via regulating 
the imbalance between Treg and Th17 cells, Mol. Med. Rep., 2017, 15(6), 3615-3622.

49 Y. Li, J. Y. Zheng, J. Q. Liu, et al., Succinate/NLRP3 Inflammasome Induces Synovial Fibroblast Activation: Therapeutical Effects of Clematichinenoside AR on Arthritis, Front. Immunol., 2016, 7, 532.

50 F. S. Mohammadi, S. Aslani, S. Mostafaei, et al., Are genetic variations in IL-21-IL-23R-IL-17A cytokine axis involved in a pathogenic pathway of rheumatoid arthritis? Bayesian hierarchical meta-analysis, J. Cell. Physiol., 2019, 234(10), 17159-17171.

51 H. Sun, H. L. Zhang, A. H. Zhang, et al., Network pharmacology combined with functional metabolomics discover bile acid metabolism as a promising target for mirabilite against colorectal cancer, $R S C A d v$., 2018, 8, 30061-30070.

52 M. F. Breed, P. A. Harrison, C. Blyth, et al., The potential of genomics for restoring ecosystems and biodiversity, Nat. Rev. Genet., 2019, DOI: 10.1038/s41576-019-0152-0.

53 A. Zhang, S. Qiu, H. Xu, et al., Metabolomics in diabetes, Clin. Chim. Acta, 2014, 429, 106-110.

54 Y. Yamanishi and G. S. Firestein, Pathogenesis of Rheumatoid Arthritis: The Role of Synoviocytes, Rheumatic Disease Clinics of North America, 2001, 27(2), 355-371.

55 J. Ren, H. Sun and H. Dong, A UPLC-MS-based metabolomics approach to reveal the attenuation mechanism of Caowu compatibility with Yunnan Baiyao, RSC Adv., 2019, 9, 8926-8933.

56 S. Konisti, S. Kiriakidis and E. M. J. N. R. R. Paleolog, Hypoxia-a key regulator of angiogenesis and inflammation in rheumatoid arthritis, Nat. Rev. Rheumatol., 2012, 8(3), 153.

57 H. Sun, F. Wu and A. Zhang, Profiling and identification of the absorbed constituents and metabolites of schisandra lignans by ultra-performance liquid chromatography coupled to mass spectrometry, Biomed. Chromatogr., 2013, 27, 1511-1519.

58 S. Cheng, C. He, H. Zhou, et al.The effect of Toll-like receptor 4 on $\beta 2$-glycoprotein I-induced $\mathrm{B}$ cell activation in mouse model, Mol. Immunol., 2016, 71, 78-86.

59 J. W. Park, H. S. Lee, Y. Lim, J. H. Paik, O. K. Kwon, J. H. Kim, I. Paryanto, P. Yunianto, S. Choi, S. R. Oh and K. S. Ahn, Rhododendron album Blume extract inhibits TNF- $\alpha /$ IFN- $\gamma$-induced chemokine production via blockade of NF- $\kappa \mathrm{B}$ and JAK/STAT activation in human epidermal keratinocytes, Int. J. Mol. Med., 2018, 41(6), 3642-3652.

60 A. Zhang, H. Sun, H. Xu, et al., Cell metabolomics, OMICS, 2013, 17, 495-501.

61 N. Liu, X. Feng, W. Wang, et al.Paeonol protects against TNF- $\alpha$-induced proliferation and cytokine release of rheumatoid arthritis fibroblast-like synoviocytes by upregulating FOXO3 through inhibition of miR-155 expression, Inflammation Res., 2017, 66(7), 1-8.

62 C. Diaz-Torne, M. D. A. Ortiz, P. Moya, et al., The combination of IL-6 and its soluble receptor is associated with the response of rheumatoid arthritis patients to tocilizumab, Semin. Arthritis Rheum., 2018, 47(6), 757-764.

$63 \mathrm{~J}$. Yang, F. Zhao and J. Nie, Anti-rheumatic effects of Aconitum leucostomum Worosch. on human fibroblastlike synoviocyte rheumatoid arthritis cells, Exp. Ther. Med., 2017, 14(1), 453-460.

64 P. Rubis, S. Wisniowska-Smialek, E. Wypasek, et al., Fibrosis of extracellular matrix is related to the duration of the disease but is unrelated to the dynamics of collagen metabolism in dilated cardiomyopathy, Inflammation Res., 2016, 65(12), 941-949.

65 H. Chen, Q. Chen, C. M. Jiang, et al., Triptolide suppresses paraquat induced idiopathic pulmonary fibrosis by inhibiting TGFB1-dependent epithelial mesenchymal transition, Toxicol. Lett., 2018, 284, 1-9.

66 X.-M. Meng, D. J. Nikolic-Paterson and H. Y. J. N. R. N. Lan, TGF- $\beta$ : the master regulator of fibrosis, Nat. Rev. Nephrol., 2016, $12(6), 325$.

67 H. Fang, A. H. Zhang, H. Sun, et al., High-throughput metabolomics screen coupled with multivariate statistical analysis identifies therapeutic targets in alcoholic liver disease rats using liquid chromatography-mass spectrometry, J. Chromatogr. B: Anal. Technol. Biomed. Life Sci., 2019, 1109, 112-120.

68 A. Iverson, E. Garza, R. Manow, et al., Engineering a synthetic anaerobic respiration for reduction of xylose to xylitol using NADH output of glucose catabolism by Escherichia coli AI21, BMC Syst. Biol., 2016, 10, 31.

69 G. M. Tannahill, A. M. Curtis, J. Adamik, E. M. PalssonMcDermott, A. F. McGettrick, G. Goel, C. Frezza, N. J. Bernard, B. Kelly, N. H. Foley, L. Zheng, A. Gardet, Z. Tong, S. S. Jany, S. C. Corr, M. Haneklaus, B. E. Caffrey, K. Pierce, S. Walmsley, F. C. Beasley, E. Cummins, V. Nizet, M. Whyte, C. T. Taylor, H. Lin, S. L. Masters, E. Gottlieb, V. P. Kelly, C. Clish, P. E. Auron, R. J. Xavier and L. A. O'Neill, Succinate is an inflammatory signal that induces IL-1 $\beta$ through HIF-1 $\alpha$, Nature, 2013, 496(7444), 238-242.

70 H. A. Elshabrawy, Z. Chen, M. V. Volin, et al., The pathogenic role of angiogenesis in rheumatoid arthritis, Angiogenesis, 2015, 18(4), 433-448.

71 Y. F. Li, S. Qiu, L. J. Gao, et al., Metabolomic estimation of the diagnosis of hepatocellular carcinoma based on ultrahigh performance liquid chromatography coupled with time-of-flight mass spectrometry, RSC Adv., 2018, 8(17), 9375-9382.

72 Z. Szekanecz and A. E. J. C. O. R. Koch, Macrophages and their products in rheumatoid arthritis, Curr. Opin. Rheumatol., 2007, 19(3), 289-295.

$73 \mathrm{~F} . \mathrm{Hu}, \mathrm{R} . \mathrm{Mu}, \mathrm{J}$. Zhu, et al., Hypoxia and hypoxia-inducible factor-1? provoke toll-like receptor signalling-induced inflammation in rheumatoid arthritis, 2014, 73(5), 928-936.

74 X. Wang, J. Li and A. H. Zhang, Urine metabolic phenotypes analysis of extrahepatic cholangiocarcinoma disease using ultra-high performance liquid chromatography-mass spectrometry, RSC Adv., 2016, 6(67), 63049-63057. 
75 I. A. Avramis, R. Kwock and V. I. J. a. R. Avramis, Taxotere and vincristine inhibit the secretion of the angiogenesis inducing vascular endothelial growth factor (VEGF) by wild-type and drug-resistant human leukemia T-cell lines, Anticancer Res., 2001, 21(4A), 2281-2286.

76 H. Du, X. Zhao, A. Zhang, et al., Identifying potential therapeutic targets of a natural product Jujuboside $\mathrm{B}$ for insomnia through network pharmacology, Plant Sci. Today, 2014, 1(2), 69-79.

77 A. Zhang, H. Sun, X. Wang, et al., Urinary metabolic profiling of rat models revealed protective function of scoparone against alcohol induced hepatotoxicity, Sci. Rep., 2014, 4, 6768.

78 Y. Li, Y. Liu, C. Wang, et al., Succinate induces synovial angiogenesis in rheumatoid arthritis through metabolic remodeling and HIF-1alpha/VEGF axis, Free Radical Biol. Med., 2018, 126, 1-14.

79 M. Ivan, K. Kondo, H. Yang, et al., HIFalpha targeted for VHL-mediated destruction by proline hydroxylation: implications for $\mathrm{O}_{2}$ sensing, Science, 2001, 292(5516), 464468.

80 A. H. Zhang, H. Sun, Y. Han, et al., Ultraperformance liquid chromatography-mass spectrometry based comprehensive metabolomics combined with pattern recognition and network analysis methods for characterization of metabolites and metabolic pathways from biological data sets, Anal. Chem., 2013, 85(15), 7606-7612.

81 Z. J. Shao, X. W. Zheng, T. Feng, et al., Andrographolide exerted its antimicrobial effects by upregulation of human beta-defensin-2 induced through p38 MAPK and NFkappaB pathway in human lung epithelial cells, Can. J. Physiol. Pharmacol., 2012, 90(5), 647-653.

82 A. Zhang, H. Sun and X. Wang, Power of metabolomics in biomarker discovery and mining mechanisms of obesity, Obes. Rev., 2013, 14(4), 344-349.

83 Q. H. Wang, S. W. Lv, Y. Y. Guo, et al., Pharmacological Effect of Caulophyllum robustum on Collagen-Induced Arthritis and Regulation of Nitric Oxide, NF- $\kappa$ B, and Proinflammatory Cytokines In vivo and In vitro, Evid.Based Complementary Altern. Med., 2017, 2017(1), 1-12.

84 X. Wang, A. Zhang, H. Sun, et al., Discovery and development of innovative drug from traditional medicine by integrated chinmedomics strategies in the postgenomic era, TrAC, Trends Anal. Chem., 2016, 76, 86-94.

85 X. Chen, X. Chen, Z. Zhou, et al., Nirogacestat suppresses RANKL-Induced osteoclast formation in vitro and attenuates LPS-Induced bone resorption in vivo, Exp. Cell Res., 2019, 111470.

86 H. Sun, A. Zhang, G. Yan, et al., Metabolomic analysis of key regulatory metabolites in hepatitis $\mathrm{C}$ virus-infected tree shrews, Mol. Cell. Proteomics, 2013, 12(3), 710-719.

87 E. Aoyama, S. Kubota, H. M. Khattab, et al., CCN2 enhances RANKL-induced osteoclast differentiation via direct binding to RANK and OPG, Bone, 2015, 73, 242-248.

88 X. Wang, A. Zhang, Y. Han, et al., Urine metabolomics analysis for biomarker discovery and detection of jaundice syndrome in patients with liver disease, Mol. Cell. Proteomics, 2012, 11(8), 370-380.

89 B. Zhou, X. Lu, Z. Tang, et al., Influence of sinomenine upon mesenchymal stem cells in osteoclastogenesis, Biomed. Pharmacother., 2017, 90, 835-841.

90 A. Zhang, H. Wang, H. Sun, et al., Metabolomics strategy reveals therapeutical assessment of limonin on nonbacterial prostatitis, Food Funct., 2015, 6(11), 3540-3549.

91 P. A. Hill, J. J. Reynolds and M. C. Meikle, Osteoblasts mediate insulin-like growth factor-I and -II stimulation of osteoclast formation and function, Endocrinology, 1995, 136(1), 124-131.

92 X. Wang, A. Zhang, G. Yan, et al., UHPLC-MS for the analytical characterization of traditional Chinese medicines, TrAC, Trends Anal. Chem., 2014, 63, 180-187.

93 Y. Aida, M. Maeno, N. Suzuki, et al., The effect of IL-1beta on the expression of matrix metalloproteinases and tissue inhibitors of matrix metalloproteinases in human chondrocytes, Life Sci., 2005, 77(25), 3210-3221.

$94 \mathrm{H}$. Fang, A. Zhang, J. Yu, et al., Insight into the metabolic mechanism of scoparone on biomarkers for inhibiting Yanghuang syndrome, Sci. Rep., 2016, 6, 37519.

95 J. L. Ren, A. H. Zhang, L. Kong, et al., Advances in mass spectrometry-based metabolomics for investigation of metabolites, RSC Adv., 2018, 8(40), 22335-22350.

96 H. Zhang, A. Zhang, X. Zhou, et al., High-throughput lipidomics reveal mirabilite regulating lipid metabolism as anticancer therapeutics, RSC Adv., 2018, 8(62), 35600-35610.

97 W. Wisitrasameewong, M. Kajiya, A. Movila, et al., DCSTAMP Is an Osteoclast Fusogen Engaged in Periodontal Bone Resorption, J. Dent. Res., 2017, 96(6), 685-693.

98 S. Qiu, A. Zhang, T. Zhang, et al., Dissect new mechanistic insights for geniposide efficacy on the hepatoprotection using multiomics approach, Oncotarget, 2017, 8(65), 108760-108770.

99 A. Zhang, H. Sun, W. Sun, et al., Trajectory analysis of metabolomics profiling in liver injured rats using ultraperformance liquid chromatography coupled with mass spectrometry, Anal. Methods, 2013, 5(19), 5294-5301.

100 L. C. Hunt, A. Upadhyay, J. A. Jazayeri, et al., An antiinflammatory role for leukemia inhibitory factor receptor signaling in regenerating skeletal muscle, Histochem. Cell Biol., 2013, 139(1), 13-34.

101 J. A. Jazayeri, N. De Weerd, W. Raye, et al., In vitro evaluation of leukemia inhibitory factor receptor antagonists as candidate therapeutics for inflammatory arthritis, J. Interferon Cytokine Res., 2007, 27(4), 281-289.

102 S. Yang, Z. Xing, T. Liu, et al., Synovial tissue quantitative proteomics analysis reveals paeoniflorin decreases LIFR and ASPN proteins in experimental rheumatoid arthritis, Drug Des., Dev. Ther., 2018, 12, 463-473.

103 M. Mellado, L. Martinez-Munoz, G. Cascio, et al., T Cell Migration in Rheumatoid Arthritis, Front. Immunol., 2015, 6, 384.

104 S. A. Morais, A. Vilas-Boas and D. A. Isenberg, B-cell survival factors in autoimmune rheumatic disorders, Ther. Adv. Musculoskeletal Dis., 2015, 7(4), 122-151. 
105 B. Nie, X. Li, Y. Wei, et al., Xianfanghuomingyin, a Chinese Compound Medicine, Modulates the Proliferation and Differentiation of $\mathrm{T}$ Lymphocyte in a Collagen-Induced Arthritis Mouse Model, Evid. Based Complementary Altern. Med., 2016, 2016, 6356871.

106 A. Zhang, H. Sun, S. Qiu, et al., Metabolomics insights into pathophysiological mechanisms of nephrology, Int. Urol. Nephrol., 2014, 46(5), 1025-1030.

107 I. M. Djuretic, D. Levanon, V. Negreanu, et al., Transcription factors T-bet and Runx3 cooperate to activate Ifng and silence Il4 in T helper type 1 cells, Nat. Immunol., 2007, 8(2), 145-153.

108 H. Sun, A. Zhang, D. Zou, et al., Metabolomics coupled with pattern recognition and pathway analysis on potential biomarkers in liver injury and hepatoprotective effects of yinchenhao, Appl. Biochem. Biotechnol., 2014, 173(4), 857869.

109 E. Bettelli, T. Korn and V. K. Kuchroo, Th17: the third member of the effector $\mathrm{T}$ cell trilogy, Curr. Opin. Immunol., 2007, 19(6), 652-657.

110 B. Astry, S. H. Venkatesha, A. Laurence, et al., Celastrol, a Chinese herbal compound, controls autoimmune inflammation by altering the balance of pathogenic and regulatory $\mathrm{T}$ cells in the target organ, Clin. Immunol., 2015, 157(2), 228-238.

111 H. Sun, L. Yang, M. Li, et al., UPLC-G2Si-HDMS untargeted metabolomics for identification of metabolic targets of YinChen-Hao-Tang used as a therapeutic agent of dampnessheat jaundice syndrome, J. Chromatogr. B: Anal. Technol. Biomed. Life Sci., 2018, 1081, 41-50.

112 H. S. Na, J. E. Kwon, S. H. Lee, et al., Th17 and IL-17 Cause Acceleration of Inflammation and Fat Loss by Inducing
alpha2-Glycoprotein 1 (AZGP1) in Rheumatoid Arthritis with High-Fat Diet, Am. J. Pathol., 2017, 187(5), 1049-1058.

113 A. Zhang, H. Sun, G. Yan, et al., Metabolomics study of type 2 diabetes using ultra-performance LC-ESI/quadrupoleTOF high-definition MS coupled with pattern recognition methods, J. Physiol. Biochem., 2014, 70(1), 117-128.

114 K. Zhang, G. Yan, A. Zhang, et al., Recent advances in pharmacokinetics approach for herbal medicine, $R S C$ Adv., 2017, 7(46), 28876-28888.

115 Y. Li, B. Yang, J. Y. Bai, et al., The roles of synovial hyperplasia, angiogenesis and osteoclastogenesis in the protective effect of apigenin on collagen-induced arthritis, Int. Immunopharmacol., 2019, 73, 362-369.

116 H. Sun, C. Liu, A. Zhang, et al., Rapid discovery and global characterization of multiple constituents from Kai-Xin-San using an integrated MS E data acquisition mode strategy based on ultra-performance liquid chromatography coupled to electrospray ionization/quadrupole-time-offlight mass spectrometry, Anal. Methods, 2015, 7(1), 279286.

117 Q. Song, A. Zhang, G. Yan, et al., Technological advances in current metabolomics and its application in tradition Chinese medicine, RSC Adv., 2017, 7(84), 53516-53524.

118 Y. Shen, B. L. Chen, Q. X. Zhang, et al., Traditional uses, secondary metabolites, and pharmacology of Celastrus species - a review, J. Ethnopharmacol., 2019, 241, 111934.

119 Q. Li, Z. Chen, Z. Xu, et al., Binding of the polysaccharide from Acanthopanax giraldii Harms to toll-like receptor 4 activates macrophages, J. Ethnopharmacol., 2019, 241, 112011. 